Canadian University Music Review

Canadian University Music Review

Revue de musique des universités canadiennes

\title{
Systèmes Musicaux en Afrique Subsaharienne
}

\section{Simha Arom}

Volume 9, numéro 1, 1988

URI : https://id.erudit.org/iderudit/1014922ar

DOI : https://doi.org/10.7202/1014922ar

Aller au sommaire du numéro

Éditeur(s)

Canadian University Music Society / Société de musique des universités

canadiennes

ISSN

0710-0353 (imprimé)

2291-2436 (numérique)

Découvrir la revue

Citer cet article

Arom, S. (1988). Systèmes Musicaux en Afrique Subsaharienne. Canadian University Music Review / Revue de musique des universités canadiennes, 9(1),

1-18. https://doi.org/10.7202/1014922ar

All Rights Reserved (C Canadian University Music Society / Société de musique des universités canadiennes, 1988
Ce document est protégé par la loi sur le droit d'auteur. L'utilisation des services d'Érudit (y compris la reproduction) est assujettie à sa politique d'utilisation que vous pouvez consulter en ligne.

https://apropos.erudit.org/fr/usagers/politique-dutilisation/ 


\title{
SYSTÈMES MUSICAUX \\ EN AFRIQUE SUBSAHARIENNE
}

\author{
Simha Arom
}

Les musiques traditionnelles africaines peuvent être caractérisées de la façon suivante :

- n'ayant pas recours à l'écriture, leur transmission s'effectue par voie orale, de bouche à oreille;

- $\quad$ populaires, elles sont dépourvues de canons explicites;

- collectives, elles appartiennent à la communauté tout entière qui est la garante de leur pérennité;

- $\quad$ anonymes et sans date, on ignore qui les a créées et quand elles ont été conçues;

- fonctionnelles - ou plus précisément, circonstanciées -, elles ne sont guère destinées à une quelconque utilisation en dehors de leur contexte socio-culturel;

- ne faisant pas l'objet de spéculations abstraites de la part de ceux qui les pratiquent, la théorie qui les sous-tend est totalement implicite. ${ }^{1}$

1 Pour ce qui est des caractéristiques générales de la musique africaine, $\mathrm{cf}$. notamment : Hornbostel 1928; Jones 1937, 1958a, 1958b et 1959; Kubik 1968; Lomax 1959; Merriam 1959 et 1962; Nketia 1972 et 1975; Rouget 1961 . 
La diversification des musiques traditionnelles coïncide avec la diversité des groupes et sous-groupes ethniques, telle qu'attestée par les langues ou dialectes qui leur sont propres. De manière générale, chaque communauté ayant recours à un dialecte, voire à un parler, qui la distingue de toutes les autres dispose, de la même façon, d'une musique qui la singularise. On peut donc considérer qu'il y a en Afrique autant de musiques que de communautés ethniques, que de langues et dialectes.

L'acquisition des bases du savoir musical va de pair avec celle du langage, c'est-à-dire qu'elle s'effectue de façon empirique. Dans une première phase, le nourrisson, passif, s'imprègne de la musique qui l'environne; au fur et à mesure que sa motricité se développe, l'enfant prendra progressivement part à diverses activités musicales, tout d'abord en frappant des mains en cadence, puis en chantant, enfin en s'essayant à un instrument. ${ }^{2}$

La cohérence des musiques traditionnelles africaines se manifeste, au sein de chaque communauté ethnique, d'une part par leur fonctionnalité - en d'autres termes, par les circonstances sociales et/ou religieuses auxquelles elles sont intimement associées -, d'autre part par leurs éléments constitutifs (instruments, échelles, structures temporelles, procédés techniques, etc.).

Il paraît important de mentionner d'emblée que, dans un même contexte culturel, fonctionnalité et systématique musicale sont étroitement liées. En effet, à chaque circonstance qui nécessite un support musical correspond un répertoire particulier, caractérisé par un nombre de chants spécifiques, une formation instrumentale prédéterminée, de même que par des structures périodiques et des formules rythmiques ou polyrythmiques qui n'appartiennent qu'à la musique associée à cette circonstance, et à elle seulement. Chaque répertoire ainsi défini correspond donc à une catégorie musicale, distincte de toutes les autres, as-

2 Concernant l'apprentissage de la musique dans les sociétés traditionnelles, cf. : Arom 1985; Basden 1921; Blacking 1957; Cudjoe 1953; Gadzekpo 1952; Gbeho 1951; John 1952; Jones 1959; Merriam 1964 et 1973; Nketia 1975. 
sociées à leur tour à telle ou telle circonstance. La totalité de la musique d'une communauté ethnique se laisse donc parfaitement classer en un ensemble fini de catégories, s'excluant mutuellement. ${ }^{3}$

Les éléments constitutifs de ces musiques font intervenir les paramètres suivants : hauteurs, temps, timbre, forme, structure et modalités d'exécution, enfin, procédés techniques. On les envisagera maintenant successivement.

L'organisation des hauteurs concerne, bien évidemment, les échelles : celles-ci vont, selon les régions et les ethnies, du système pentatonique anhémitonique jusqu'à l'échelle équiheptatonique, en passant par le pentatonisme fondé sur des intervalles de tons entiers, l'échelle diatonique, ou encore celles présentant des degrés mobiles selon que la courbe mélodique est ascendante ou descendante. Dans bien des cas, une même communauté fait appel à divers types d'échelles, qui sont alors vouées à des répertoires différents. ${ }^{4}$

La structuration du temps a pour base une musique qui est pratiquement toujours mesurée, c'est-à-dire au sein de laquelle les durées entretiennent des rapports strictement proportionnels.

La grande majorité des musiques africaines est fondée sur une périodicité rigoureuse, déterminée par le retour d'événements semblables en des positions identiques d'un cycle temporal. Toute période se subdivise en un nombre constant de pulsations isochrones - c'est-à-dire équidistantes - qui constituent l'étalon organique par rapport auquel s'ordonnent toutes les durées, tant à l'horizontale - lorsqu'il s'agit de

3 À ce sujet, cf. : Arom 1973, 1974, 1976, 1981a, 1984a et 1985; Dehoux 1986; Joyeux 1924; Merriam 1962 et 1964; Nketia 1975; Reich 1974; Schaeffner 1936 et 1960; Schmidt-Wrenger 1975.

4 Pour ce qui est des différents types d'échelle, cf. notamment : Ballantine 1965; Berliner 1978; Brandel 1970; Gide 1928; Grimaud 1957 et 1960; Kirby 1934 et 1952; Kolinski 1960; Kubik 1961, 1964a, 1968 et 1969; Merriam 1959; Nketia 1972 et 1975; Rouget 1961 b et 1969; Tracey 1958 et 1969. 
monodie - qu'à la verticale, au sein des édifices polyphoniques et/ou polyrythmiques.

On aura noté qu'une telle organisation du temps diffère considérablement de la conception occidentale, en ce qu'elle fait abstraction, le plus souvent, du niveau intermédiaire de la "mesure", c'est-à-dire d'un schéma accentuel régulier fondé sur l'alternance d'un temps fort avec un ou plusieurs temps faibles.

La pulsation se subdivise à son tour en valeurs plus brèves, selon un principe binaire ou ternaire, mais parfois aussi en associant les deux, ce qui donne lieu à des combinaisons de $2+3$ ou $3+2$. Ce sont là les valeurs opérationnelles minimales par le biais desquelles toutes les durées et les rapports de durées différentes superposées vont pouvoir s'ordonner. 5

Le timbre concerne les "outils" qui concourent à la matérialisation de la musique, c'est-à-dire la voix et les instruments.

Certains répertoires ne font appel qu'à des voix d'hommes, d'autres exclusivement à des voix de femmes, d'autres encore sont réservés aux seuls enfants.

La combinaison des timbres instrumentaux peut se résumer à trois catégories, qui apparaissent ici selon l'ordre décroissant de leur fréquence d'utilisation :

5 Pour ce qui est de la structuration du temps, cf. : Akpabot 1971; Arom 1973, 1976, 1984a, 1984b et 1985; Ballantine 1965; Béclard-d'Harcourt 1924; Berliner 1978; Blacking 1961 et 1971; Dauer 1966; Dehoux 1986; England 1967; Estreicher 1957 et 1964; Golberry 1802; Harrison 1973; Jones 1937, 1954, 1958a, 1958b et 1959; Jones et Kombe 1952; Kirby 1934 et 1936; Koetting 1970; Kolbe 1741; Kolinski 1960 et 1973; Kubik 1961, 1964a et 1964b; Locke 1982; Lomax 1968; Merriam 1962; Nketia 1963 et 1975; Pantaleoni 1972; Park 1800; Reich 1974; Rouget 1961b; Rycroft 1967; Schmidt-Wrenger 1975; Waterman 1952. 
- des instruments de type différent jouant ensemble (par exemple, un xylophone dont l'accompagnement rythmique est confié à un tambour, un hochet et une poutrelle frappée);

- différents instruments de même type (comme deux ou trois xylophones) sont supportés par un ou plusieurs instruments à percussion différents (comme précédemment, par exemple);

- enfin, des formations où tous les instruments sont de même type : c'est le cas des orchestres de trompes ou de sifflets, qui peuvent regrouper jusqu'à une vingtaine d'instruments.

Rappelons qu'en milieu traditionnel, la formation instrumentale c'est-à-dire sa composition spécifique sous l'angle des instruments qui la constituent - est indissociable de telle ou telle circonstance.

Quant aux formes et structures, il convient de faire une premièe distinction entre celles qui n'admettent aucune modification en cours d'exécution et celles qui se renouvellent à chaque execution. Les premières, extrêmement rares, sont presque toujours liées à l'évocation d'événements à caractère historique ou bien font partie d'importantes liturgies. Ce sont là des musiques figées, par opposition à la très grande majorité où, ou sein de structures périodiques, métriques et rythmiques extrêmement rigoureuses, une part de liberté est laissée aux exécutants, leur permettant ainsi la réalisation de nombreuses variations, souvent même de véritables improvisations. ${ }^{6}$

Une seconde distinction s'impose entre les musiques qui sont strictement cycliques - c'est-à-dire répétitives -, que l'on peut qualifier de "fermées" et celles qui, par opposition, peuvent être dites "ouvertes" en ce que leur déroulement est fondé sur l'alternance irrégulière de diverses

6 Concernant les formes, structures, patterns, cf. : Agawu 1986; Akpabot 1971; Arom 1973, 1976, 1981a, 1984a, 1984b, 1984c et 1985; Berliner 1978; Blacking 1961; Dehoux 1986; Ekwueme 1975; Jones 1937, 1958b et 1959; Kauffman 1980; King 1960; Kirby 1934; Koetting 1970; Kubik 1961 et 1968; Lomax 1968; Merriam 1962; Nketia 1962, 1967, 1972 et 1975; Reich 1974; Rouget 1956 et 1961 b; Schaeffner 1936 et 1960; Schmidt-Wrenger 1975. 
structures périodiques juxtaposées, donnant lieu ainsi à des oeuvres de bien plus grande envergure et souvent d'un grand souffle. Mais qu'elles soient ouvertes ou fermées, ces musiques - hormis quelques rares exceptions - laissent toujours à ceux qui les pratiquent une vaste marge de liberté où leur créativité spontanée peut s'exprimer. ${ }^{7}$

Les modalités d'exécution concernent la répartition des rôles au sein de l'événement musical, notamment entre soliste vocal et choeur ou, lorsqu'un chanteur s'accompagne lui-même d'un instrument, entre partie vocale et instrumentale.

Dans le premier cas, la situation la plus fréquente est celle d'une alternance régulière entre les deux protagonistes : soit que le choeur reprend textuellement la phrase énoncée par le soliste - il s'agit alors d'un chant de type antiphonal —, soit que le choeur complète celle-ci, ce qui confère à la musique un caractère responsorial.

De manière générale, le chanteur qui s'accompagne lui-même d'un instrument mélodique, divise, lui aussi, la période musicale en deux segments, l'un confié à sa propre voix - que l'instrument soutient, naturellement - , l'autre consistant en un répons de l'instrument. Ainsi s'instaure un dialogue permanent entre ces deux éléments, insufflés par un seul et même esprit.

Une telle répartition des rôles n'est pas le fait du hasard. Elle procède, bien au contraire, d'une parfaite logique. Attestant une segmentation cohérente du discours musical, les modalités d'exécution constituent un outil important pour la découverte des structures qui le sous-tendent.

7 Au sujet de la variation, cf. : Arom 1976 et 1985; Ballantine 1965; Belinga 1965; Blacking 1971; Brandel 1961 et 1970; Dauer 1966; England 1967; Estreicher 1964; Godsey 1984; Grimaud 1960; Gunther 1964; Hood 1971; Jones 1937, 1954, 1958a, 1958b et 1959; Kirby 1934; Koetting 1970 et 1984; Kubik 1961, 1964a et 1964b; Merriam 1959 et 1964; Nketia 1962 et 1975; Pantaleoni 1972. 
Dans les procédés techniques, il y a lieu de distinguer entre l'usage de la monodie et celui de la polyphonie.

La monodie peut être vocale et/ou instrumentale. Lorsque le chant est redoublé par un instrument mélodique, il est fréquent que la coïncidence mélodique ou rythmique ne soit pas absolue entre les deux. Ce phénomène - souvent volontaire - donne alors lieu à la forme la plus rudimentaire de polyphonie, l'hétérophonie.

La polyphonie à proprement parler a recours, le plus souvent, à l'une des techniques suivantes :

- le mouvement parallèle des parties, par quintes et quartes ou sixtes et tierces. Ce procédé est extrêmement répandu dans les musiques vocales;

- le mouvement divergent, qui peut être de type homophone lorsque l'articulation rythmique est la même pour toutes les parties en présence - ou contrapuntique - lorsque les parties sont rythmiquement indépendantes. L'emploi du contrepoint est fort rare dans la musique vocale (deux populations seulement, à notre connaissance, le pratiquent : les Pygmées d'Afrique Centrale et les Boshiman du désert du Kalahari). En revanche, ce type de polyphonie est relativement fréquent dans la musique instrumentale; 8

- la technique du hoquet, propagée de l'Éthiopie jusqu'à l'Afrique du Sud, en passant par l'Afrique de l'Ouest, de l'Est et du Centre. Essentiellement instrumentale, elle est en premier lieu le fait de

8 Pour la polyphonie en général, cf. : Akpabot 1971; Arom 1973, 1976, 1981b et 1985; Bosman 1705; Bowdich 1819; Brandel 1952, 1961, 1965, et 1970; Collaer 1954; Dauer 1969; Dehoux 1986; England 1967; Gide 1927 et 1928; Golberry 1802; Grimaud 1957, 1960 et 1964; Gunther 1964; Haardt et Audouin 1927; Hornbostel 1928; Hornburg 1950; Joyeux 1910 et 1924; Kauffman 1984; Kirby 1930, 1933, 1934 et 1936; Knight 1984; Kubik 1961, 1964a, 1965 et 1968; Labat 1732; Lomax 1959; Mensah 1967; Nketia 1967, 1972 et 1975; Pantaleoni 1972; Rouget 1961a et 1961b; Rycroft 1967; Schaeffner 1936, 1950 et 1951; Schweinfurth 1875; Wachsmann 1953. 
formations faisant appel à des instruments de même type (trompes ou sifflets) dont chacun ne peut émettre qu'un seul et même son. ${ }^{9}$

- le dernier - mais non le moindre - procédé qu'il convient de mentionner ici est la polyrythmie. La plus grande partie des musiques traditionnelles africaines est supportée par des ensembles d'instruments à percussion, au sein desquels le paramètre mélodique est neutralisé.

Fournissant le cadre périodique et l'armature métrique aux événements mélodiques qui s'y superposent, la musique que ces instruments réalisent est fondée sur l'entrecroisement des diverses figures rythmiques propres à chacun d'eux, et dont les accents sont décalés, ce qui a pour effet de produire un enchevêtrement extrêmement dense - mais toujours cohérent - de rythmes antagonistes et, par là même, une polyrythmie d'une trame aussi touffue que complexe. ${ }^{10}$

Parmi tous ces paramètres, chaque aire géo-culturelle, mais souvent aussi chaque groupe ethnique, voire sous-groupe, opère une sélection, afin de les combiner selon des choix propres qui sont les siens.

C'est ainsi que s'explique l'extraordinaire richesse et la très grande variété des musiques que l'on rencontre sur le continent africain.

9 Concernant la technique du hoquet, cf. : Arom 1973, 1976, 1981a, 1984d et 1985; Ballantine 1965; Brandel 1965 et 1970; Gide 1927; Herzog 1949; Joyeux 1924; Kirby 1934; Koetting 1984; Mensah 1967; Nketia 1962, 1972 et 1975; Schaeffner 1951; Schmidt 1984.

10 Au sujet de la polyrythmie, cf. : Agawu 1986; Akpabot 1971; Arom 1973, 1976, 1981b, 1984b, 1984c et 1985; Ballantine 1965; Béclard-d'Harcourt 1924; Blacking 1961; Brandel 1952, 1961 et 1970; Dauer 1966 et 1969; England 1967; Estreicher 1964; Grimaud 1960; Gunther 1964; Hood 1971; Jones 1934, 1937, 1954, 1958a et 1959; Kauffman 1980; Kirby 1934 et 1936; Koetting 1970; Kolinski 1973; Kubik 1961, 1962 et 1964a; Lomax 1968; Merriam 1959; Nketia 1962, 1972 et 1975; Pantaleoni 1972; Reich 1974; Rouget 1961b; Sachs 1943,1953 et 1962 . 
Ainsi, bien que les dépositaires de ces musiques n'en fassent pas un object de spéculations abstraites, il n'en demeure pas moins que tout idiome musical traditionnel en Afrique, en ce qu'il obéit toujours à un canon de règles, relève bel et bien d'une théorie - même si celle-ci est implicite - et constitue par conséquent un système au plein sens du terme. 


\section{RÉFÉRENCES}

AGAWU, V. Kofi

1986 : “Gi Dunu', 'Nyekpadudo', and the Study of West African Rhythm", Ethnomusicology XXX/1 : 64-81.

AKPABOT, Samuel

1971 : “African Instrumental Music”, African Arts, V/1 : 63-64.

AROM, Simha

1973 : "Une méthode pour la transcription des polyphonies et polyrythmies de tradition orale", Revue de Musicologie LIX/2 : 165-190.

1974 : "De la chasse au piège considérée comme une liturgie", The World of Music XVI/4 : 3-19.

1976 : "The Use of Play-back Techniques in the Study of Oral Polyphonies", Ethnomusicology XX/3 : 483-519.

1978 : "Rythmique et polyrythmie centrafricaines : fonctions, structure", Conférences des Journées d'Étude, Festival International du Son, Paris, Editions Radio : 163-172.

1981a : "New Perspectives for the Description of Orally Transmitted Music", The World of Music XXIII/2 : 40-60.

1981b : "Central African Republic", The New Grove's Dictionary of Music and Musicians, London: McMillan, Vol. 3 : 57-62.

1984a : "Comprendre la musique des Autres", Recherche, Pédagogie et Culture, LXV-LXVI : 29-34.

1984b : "The Constituting Features of Central African Rhythmic Systems. A Tentative Typology", The World of Music XXIV/1 : 51-67.

1984c : "Structuration du temps dans les musiques d'Afrique Centrale : périodicité, mètre, rythme et polyrythmie", Revue de Musicologie LXX/1 : 5-36.

1984d : "The Music of Banda-Linda Horn Ensembles. Form and Structure", Selected Reports in Ethnomusicology, Vol. V : Studies in African Music, Los Angeles: University of California : 173-193.

1985 : Polyphonies et polyrythmies d'Afrique Centrale : structure et méthodologie. Paris : SELAF, 2 volumes. 
BALLANTINE, Christopher

1965 : "The Polyrhythmic Foundation of Tswana Pipe Melody", African Music, III/4 : 52-67.

BASDEN, G. T.

1921 : Among the Ibos of Nigeria. Philadephia : J. B. Lippincott.

BECLARD-D'HARCOURT, Marguerite

1924 : "Notes relatives à la transcription des phonogrammes", in Joyeux, Ch. : "Etude sur quelques manifestations musicales observées en Haute-Guinée Française", Revue d'Ethnographie et des Traditions Populaires, V/18 : 173-202.

BELINGA, M. S. Eno

1965 : Littérature et musique populaire en Afrique Noire. Paris : Cujas.

BERLINER, Paul F.

1978 : The Soul of Mbira. Los Angeles : University of California Press.

BLACKING, John

1957 : The Role of Music amongst the Venda of Northern Transvaal. Johannesburg : International Library of African Music.

1961 : "Patterns of Nsenga Kalimba Music", African Music, II/4 : 26-43.

1971 : "Deep and Surface Structure in Venda Music", Yearbook of the International Folk Music Council III : 91-108.

BOSMAN, Guillaume

1705 : Voyage de Guinée, contenant une Description nouvelle et très exacte de cette côte[...], Utrecht : Antoine Schouten.

BOWDICH, Thomas Edward

1819 : Mission from Cape Coast to Ashantee. London : John Murray.

BRANDEL, Rose

1952 : "Music of the Giants and the Pygmies of the Belgian Congo", Journal of the American Musicological Society V/1 : 16-28.

1961 : The Music of Central Africa. An Ethnomusicological Study. The Hague : Martinus Nijhoff.

1965 : "Polyphony in African Music", The Commonwealth of Music in Honour of Curt Sachs, New York : The Free Press : 26-44. 
1970 : "Africa”, Harvard Dictionary of Music, 2nd ed. : 17-24.

COLLAER, Paul

1954 : "Notes sur la musique d'Afrique Centrale", Problèmes d'Afrique Centrale XXVI : 267-271.

CUDJOE, S. D.

1953 : "The Techniques of Ewe Drumming and the Social Importance of Music in Africa", Phylon XIV : 280-291.

DAUER, A. M.

1966 : "Afrikanische Musik und völkerkundlicher Tonfilm. Ein Beitrag zur Methodik der Transkription", Research Film V/5 : 439-456.

1969 : "Research Films in Ethnomusicology : Aims and Achievements", Yearbook of the International Folk Music Council I : 226-231.

DEHOUX, Vincent

1986 : Les "chants à penser" des Gbaya de Centrafrique. Paris : SELAF.

EKWUEME, Lazarus

1975-76 : "Structural Levels of Rhythm and Form in African Music", African Music V/4 : 27-35.

ENGLAND, Nicholas $M$.

1967 : "Bushman Counterpoint", Journal of the International Folk Music Council XIX : 58-66.

ESTREICHER, Zygmunt

1957 : "Une technique de transcription de la musique exotique," Bibliothèques et Musées de la Ville de Neuchâtel (Rapport), Neuchâtel : 67-92.

1964 : "Le rythme des Peuls Bororo", Les Colloques de Wégimont IV, Paris, "Les Belles Lettres", Bibliothèque de la Faculté de Philosophie et Lettres de l'Université de Liège, Fasc. CLXXII : 185-228.

GADZEKPO, Sinedzi B.

1952 : "Making Music in Eweland", West African Review XXIII : 817-821.

GBEHO, Philip

1951 : "Beat of the Master Drum" West African Review XXII : 1263-1265.

GIDE, André

1927 : Voyage au Congo. Carnets de route. Paris : Gallimard.

1928 : Le retour du Tchad. Carnets de route. Paris : Gallimard. 
GODSEY, Larry

1984 : "The Use of Variation in Birifor Funeral Music", Selected Reports in Ethnomusicology, Vol. V : Studies in African Music, Los Angeles : University of California Press : 67-80.

GOLBERRY, Sylvain Meinrad Xavier de

1802 : Fragments d'un voyage en Afrique[...], Paris : 2 volumes.

GRIMAUD, Yvette

1960 : "Note sur la musique vocale des Bochiman !Kung et des Pygmées Babinga", Les Colloques de Wégimont III, Paris, "Les Belles Lettres" : 105-126.

1964 : 'Etude analytique de la danse 'Choma' des Bochiman !Kung (polyrythmie)", Les Colloques de Wégimont 1958-1960, Paris, "Les Belles Lettres", Bibl. de la Fac. de Philo. et Lettres de Liège, Fasc. CLXXII : 171-183.

GRIMAUD, Yvette (avec la collaboration de Gilbert ROUGET)

1957 : Note sur la musique des Bochiman comparée à celle des Pygmées Babinga[...], Cambridge (USA) et Paris, Peabody Museum : Harvard Museum et Musée de l'Homme.

GUNTHER, Robert

1964 : Musik in Rwanda. Ein Beitrag zur Musikethnologie Zentral-Afrikas, Tervuren : Annales, Sciences Humaines no 50.

HAARDT, Georges-Marie et AUDOUIN-DUBREUIL, Louis

1927 : La croisière noire. Expédition Citroën Centre-Afrique. Paris : Plon.

HARRISON, Frank

1973 : Time, Place and Music: An Anthology of Ethnomusicological Observation c. 1500 to c. 1800 . Amsterdam : Frits Knuf.

HOOD, Mantle

1971 : The Ethnomusicologist. New York : McGraw-Hill.

HORNBOSTEL, Erich M. von

1928 : "African Negro Music", Africa, Journal of the International Institute of African Languages and Culture $\mathrm{I} / 1$ : 30-62.

HORNBURG, Friedrich

1950 : "Phonographierte Afrikanische Mehrstimmigkeit", Die Musikforschung III/2 : 120-142 et 161-176.

JOHN, J.T.

1952 : "Village Music of Sierra Leone", West African Review XXIII : 1043-1045, 1071 . 
JONES, A.M.

1934 : "African Drumming. A study of the Combination of Rhythms in African Music", Bantu Studies VIII/1 : 1-16.

1937 : "The Study of African Musical Rhythms", Bantu Studies XI : 295-319.

1954 : "African Rhythm, Africa", Journal of the International African Institute XXIV : 26-47.

1958a : African Music in Northern Rhodesia and Some Other Places. Livingstone : The Rhodes-Livingstone Museum.

1958b : "On Transcribing African Music", African Music II/1 : 11-14.

1959 : Studies in African Music. London : Oxford University Press.

JONES, A.M. et KOMBE, L.

1952 : The Icila Dance Old Style. A Study in African Music and Dance of the Lala Tribe of Northern Rhodesia. Cape Town : African Music Society.

JOYEUX, Charles

1910 : "Notes sur quelques manifestations musicales observées en Haute-Guinée", La Revue Musicale X/2 : 49-58.

1924 : "Etude sur quelques manifestations musicales observées en Haute-Guinée Française", Revue d'Ethnographie et des Traditions Populaires V/18 : 170-212.

KAUFFMAN, Robert

1980 : "African Rhythm : A Reassessment”, Ethnomusicology XXIV/3 : 393-415.

1984 : "Multipart Relationships in Shona Vocal Music", Selected Reports in Ethnomusicology, Vol. V : Studies in African Music, Los Angeles : University of California Press : 145-160.

KING, Anthony

1960 : "Employments of the 'Standard Pattern' in Yoruba Music", African Music II/3 : 51-54.

KIRBY, Percival R.

1930 : “A Study of Negro Harmony”, The Musical Quarterly XVI/3 : 404-430.

1933 : "The Reed-Flutes Ensembles of South Africa : A study in South African Native Music", Journal of the Royal Anthropological Institute of Great Britain and Ireland LXIII : 313-388.

1934 : The Musical Instruments of the Native Races of South Africa. London : Oxford University Press. 
1936 : "The Musical Practices of the Auni and $\neq$ Khomani Bushmen", Bantu Studies X : 373-431.

1952 : "Buschmann und Hottentotten", Die Musik in Geschichte und Gegenwart, Kassel : Bärenreiter, Vol. II : 502-510.

KNIGHT, Roderic

1984 : "The Style of Mandinka Music : A Study in Extracting Theory from Practice", Selected Reports in Ethnomusicology, Vol V : Studies in African Music, Los Angeles : University of California : 3-66.

KOETTING, James

1970 : "Analysis and Notation of West African Drum Ensemble Music", Selected Reports in Ethnomusicology, Vol I/3, Los Angeles : University of California : 116-146.

1984 : "Hocket Concept and Structure in Kasena Flute Ensemble Music", Selected Reports in Ethnomusicology, Vol. V : Studies in African Music : 161-172.

1986 : "What Do We Know About African Rhythm?", Ethnomusicology $\mathrm{XXX} / 1$ : 58-63.

KOLBE, Pierre

1741 : Description du Cap de Bonne-Espérance[...], Amsterdam : Jean Catuffe.

KOLINSKI, Mieczyslaw

1960 : "Compte rendu de A.M. JONES : Studies in African Music", The Musical Quarterly XVI/1 : 105-110.

1973 : "A Cross-Cultural Approach to Metro-Rhythmic Patterns", Ethnomusicology XVII/3 : 494-506.

KUBIK, Gerhard

1961 : "Musikgestaltung in Afrika", Neues Afrika V : 195-200.

1962 : "Oral Notation of Some West and Centrai African Time-Line Patterns", Review of Ethnology III/2 : 169-176.

1964a : "Harp Music of the Azande and Related Peoples in the Central African Republic[...]", African Music III/3 : 37-76.

1964b : "Xylophone Playing in Southern Uganda", The Journal of the Royal Anthropological Institute XCIV/2 : 138-159.

1965 : "Transcription of Mangwilo Xylophone Music from Film Strips", African Music III/4 : 35-51.

1968 : Mehrstimmigkeit und Tonsysteme in Zentral- und Ostafrika[...], Wien : Böhlaus. 
1969 : "Transmission et transcription des éléments de musique instrumentale africaine", Bulletin of the International Committee on Urgent Anthropology and Ethnological Research XI : 47-61.

LABAT, Jean-Baptiste

1732 : Relation historique de l'Éthiopie occidentale[...], Paris : C.J.B. Delespine le fils.

LOCKE, David

1982 : "Principles of Offbeat Timing and Cross-Rhythm in Southern Ewe Dance Drumming", Ethnomusicology XXVI/2 : 217-246.

LOMAX, Alan

1959 : "Folk Song Style", American Anthropologist LXI/6 : 927-954.

1968 : Folk Song Style and Culturel...], Washington D.C. : American Association for the Advancement of Science (Publication No. 88).

MENSAH, Atta A.

1967 : "The Polyphony of Gyil-gu, Kudzo an Awutu Sakumo", Journal of the International Folk Music Council XIX : 75-79.

MERRIAM, Alan P.

1959 : "Characteristics of African Music", Journal of the International Folk Music Council XI : 13-18.

1962 : "The African Idiom in Music", Journal of American Folklore LXXV/2 : 120-130.

1964 : The Anthropology of Music. Evanston : Northwestern University Press.

1973 : "The Bala Musician", The Traditional Artist in African Society, Bloomington : Indiana University Press : 250-281.

NKETIA, J.H. Kwabena

1954 : "The Role of the Drummer in Akan Society", African Music I : 34-43.

1962 : "The Hocket-Technique in African Music", Journal of the International Folk Music Council XIV : 44-52.

1963 : Folk Songs of Ghana. Legon : University of Ghana.

1967 : "Multi-part Organization in the Music of the Gogo of Tanzania", Journal of the International Folk Music Council XIX : 79-88.

1972 : "Les langages musicaux de l'Afrique Subsaharienne. Etude Comparative", La musique africaine. Réunion de Yaoundé[...], La Revue Musicale CCLXXXVIII-IX : 7-42. 
1973 : "The musician in Akan Society", The Traditional Artist in African Society, Bloomington : Indiana University Press : 79-100.

1975 : The Music of Africa. London : Victor Gollancz.

PANTALEONI, Hewitt

1972 : "The Three Principles of Timing in Anlō Dancing Drumming", African Music V/2 : 50-57.

PARK, Mungo

1800 : Voyage dans l'intérieur de l'Afrique[...]. Paris : Dentu et Casteret, 2 volumes.

REICH, Steve

1974 : "Gahu, A Dance of the Ewe Tribe in Ghana", Writings about Music, New York : New York University Press.

ROUGET, Gilbert

1956 : "Å propos de la forme dans les musiques de tradition orale", Les Colloques de Wégimont, Bruxelles : Elsevier : 132-144.

1961a : "La musique en Afrique Noire", Enc. Fasquelle, Paris, Fasquelle, Vol. III : 939-940.

1961b : "Un chromatisme africain", L'Homme. Revue Française d'Anthropologie I/3 : 1-15.

ROUGET, Gilbert (avec la collaboration de Jean SCHWAEZ)

1969 : "Sur les xylophones équiheptaphoniques des Malinké", Revue de Musicologie LV/1 : 47-77.

RYCROFT, David

1967 : "Nguni Vocal Polyphony", Journal of the International Folk Music Council XIX : 88-103.

SACHS, Curt

1943 : The Rise of Music in the Ancient World East and West. New York :W.W. Norton.

1953 : Rhythm and Tempo. A Study in Music History. New York : W.W. Norton.

1962 : The Wellsprings of Music. Amsterdam : Martinus Nijhoff.

SCHAEFFNER, André

1936 : Origine des instruments de musique. Introduction ethnologique à l'histoire de la musique instrumentale. Paris : Payot.

1950 : "La découverte de la musique noire", Le Monde Noir, numéro spécial VIII-IX : 205-218. 
1951 : Les Kissi, une société noire et ses instruments de musique. Paris : Hermann.

1960 : "Situation des musiciens dans trois sociétés africaines", Les Colloques de Wégimont III, Paris : Société d'édition "Les Belles Lettres" : 33-49.

SCHMIDT, Cynthia E.

1984 : "Interlocking Techniques in Kpelle Music", Selected Reports in Ethnomusicology, Vol. V : Studies in African Music 195-216.

SCHMIDT-WRENGER, Barbara

1975 : Musique des Tshokwe du Zaïre (brochure accompagnant le disque qui porte le même titre), Coll. "Enregistrements de musique africaine", Tervuren : Musée Royal d'Afrique Centrale, No 11.

SCHWEINFURTH, Georg

1875 : Au coeur de l'Afrique 1868-1871[...], Paris : Hachette, 2 volumes.

TRACEY, Hugh

1958 : "Towards an Assessment of African Scales", African Music II/1 : 10-20.

1969 : "Measuring African Scales", African Music IV/3 : 73-77.

WACHSMANN, K.P.

1953 : Tribal Crafts of Uganda. Part Two : The Sound Instruments. London : London University Press.

WATERMAN, Richard Alan

1952 : "African Influence on the Music of the Americas", Acculturation in the Americas, Chicago : The University of Chicago Press. 